\title{
Relationship between serum homocysteine level and cognitive impairment in patients with Parkinson's disease
}

https://doi.org/10.1515/pteridines-2019-0023

received April 19, 2019; accepted November 6, 2019.

\begin{abstract}
OBJECTIVE To investigate the correlation between serum homocysteine (Hcy) and cognitive impairment (CI) in patients with Parkinson's disease (PD).

METHODS Eighty-one PD patients were prospectively recruited in this study from Feb 2015 to Jan 2018 in Gansu Provincial Hospital. Of the subjects, 41 were diagnosed with cognitive impairment (PD-CI) vs. the 40 others without PD (PDN). The clinical characteristic and demographic features were recorded for the two groups. The serum Hcy, folate and vitamin B12 (VitB12) were examined by high-performance liquid chromatography (HPLC) and enzyme-linked immunosorbent assay (ELISA). RESULTS The serum Hcy, folate, VitB12 concentration were $21.7 \pm 6.2(\mu \mathrm{mol} / \mathrm{L}), 9.2 \pm 3.7(\mathrm{ng} / \mathrm{mL}), 354.1 \pm 123.5(\mathrm{pg} /$ $\mathrm{mL})$ for PD-CI group and 14.1 $\pm 5.7(\mu \mathrm{mol} / \mathrm{L}), 12.4 \pm 4.5(\mathrm{ng} /$ $\mathrm{mL}), 378.7 \pm 128.2(\mathrm{pg} / \mathrm{mL})$ for $\mathrm{PDN}$ group respectively. The serum level of Hcy in PD-CI group was significantly higher than that of PDN group $(p<0.05)$, serum folate was significantly lower than PDN group $(\mathrm{p}<0.05)$. The diagnostic sensitivity, specificity and AUC were 77.5\% (95\%CI:61.6\%-89.2\% ), 78.1\% (95\%CI:62.4\%-89.4\%), 0.82 (95\%CI:0.73-0.91) for serum Hcy and 72.5\% (95\%CI:56.1\%85.4\%), 63.4\% (95\%CI:46.9\%-77.9\%), 0.71(95\%CI:00600.83 ) for serum folate respectively as serological markers for cognitive impairment diagnosis in patients with PD. Conclusion Serum Hcy and folate were different between PD-CI and PDN patients, which may play an important role in cognitive impairment development in patients with
\end{abstract}

*Corresponding author: Yi Zhang, Department of Neurology, Gansu Provincial Hospital 730000 PR China, E-mail: zhangyi9310@21cn.com Xuejuan Liu, Yumei Zhao, Jingwen Yang, Taowen Ren, Baiyu Li, Lijuan Bao, Keping Jiao, Department of Neurology Cadre Ward, Gansu Provincial Hospital 730000 PR China

Tong Dong, Cheng Gu, Yamin Zhang, Department of Neurology, Gansu Provincial Hospital 730000 PR China

\# Equal contributor:Xuejuan Liu and Tong Dong contributed equally to this work.
PD and can be used as promising serological diagnostic marker.

Keywords: Parkinson's disease; cognitive impairment; Hcy; folate; serological marker.

\section{Introduction}

Parkinson's disease (PD) is a common degenerative disease of the nervous system. It is common in the elderly $[1,2]$. The average age of onset is about 60 years old, and it is rare in young people under 40 years. The prevalence of PD among people over 65 years old in China is about $1.7 \%$ [3]. Most patients with PD are sporadic cases, and fewer than $10 \%$ of them have family history [4]. The main pathological change of PD is the degeneration of dopamine (DA) neurons in the substantia nigra of the midbrain, which results in a significant decrease in DA content in the striatum [5, 6]. The exact cause of this pathological change is still unclear. Genetic and environmental factors, as well as aging, may be involved in the process of PD [7].

Cognitive impairment (CI) is one of the most common and disabling non-motor symptoms of PD [8]. The incidence of mild cognitive dysfunction can reach 20\%-40\% in newly diagnosed PD patients. After 3-5 years of follow-up, $80 \%$ of PD mild CI patients will develop Parkinson's dementia [9]. Studies have shown that $22 \%$ of PD mild CI patients can recover to normal, indicating that the pathological process of PD cognitive impairment was reversible [10]. Therefore, it is important to identify biomarkers for CI of Parkinson's patients in the early stage.

Homocysteine (Hcy) is toxic to neurons and vascular endothelial cells, and known as an independent risk factor for dementia and cardiovascular disease [11]. However, the correlation between serum level of Hcy and $\mathrm{CI}$ in patients with $\mathrm{PD}$ remained unclear. 


\section{Material and methods}

\section{Patients recruitment}

Eighty-one hospitalized PD patients were prospectively recruited in this study from Feb 2015 to Jan 2018 in Gansu Provincial Hospital. Of the subjects, 41 were diagnosed of mild cognitive impairment (PD-CI) and the other 40 cases were not (PDN). All the patients received levodopa (LED) for treatment. The project was approved by the Medical Ethics Committee of Gansu Provincial Hospital. The subject inclusion criteria were: (1) age ranges from 40 to 80; (2) the PD diagnosis was performed by two neurologists according to United Kingdom PD Society Brain Bank and Gelb's criteria [12, 13]; (3) written informed consent was obtained. The exclusion criteria were: (1) Alzheimer's disease and etc. which can cause cognitive impairment; (2) Secondary PD caused by trauma, cerebrovascular, drug and other inducements; (3) Relevant disease such as malnutrition, thyroid insufficiency, gout, malignant tumors, etc., which can cause abnormal folic acid metabolism; (4) Renal dysfunction subjects.

Informed consent: Informed consent has been obtained from all individuals included in this study

Ethical approval: The research related to human use has been complied with all the relevant national regulations, institutional policies and in accordance the tenets of the Helsinki Declaration, and has been approved by the Medical Ethics Committee of Gansu Provincial Hospital.

\section{Clinical characteristic and demographic features collection}

Two experienced neurophysicians collected the detailed demographic data (age, gender, diabetes, education level, etc.), course of disease and treatment methods. The data were cross-checked in order to ensure accuracy.

\section{MoCA-score and UPDRS III-score measure measurement}

All subjects were assessed by two neurologists trained in professional scales under quiet conditions. MoCA and UPDRSIII were used to evaluate the cognitive function of all subjects. The evaluation was carried out according to the instructions and steps of the scale. MoCA is a screening instrument composed of eight sections including visuospatial/executive, naming, memory, attention, language, abstraction, delayed recall, and orientation with a total score of 30. UPDRS is mainly for motor function examination, the total score is 108 which indicates the higher the score, the more serious the motor function injury is.

\section{Serum Hcy, folate, VitB12 detection}

Five $\mathrm{ml}$ of fasting venous blood was collected from all subjects in the morning and processed within $30 \mathrm{~min}$. The fresh serum was collected at room temperature of 3000 $\times g$ and centrifuged for $5 \mathrm{~min}$. The fresh serum was stored at -80 al. The serum Hcy, folate and VitB12 were examined by high-performance liquid chromatography (HPLC) and enzyme-linked immunosorbent assay (ELISA). All the examination procedure was done according to the instructions for kit operation.

\section{Statistical analysis}

All the data were analyzed by SPSS 18.0 statistical software package (IBM, Armonk, NY, USA). Serum Hcy, folate, VitB12, etc. were expressed as mean \pm standard deviation. The comparison between PD-CI and PDN groups was done by student-t test. Chi-square test was used for comparison of categorical data. Significance of serum Hcy, folate as a biomarker in the diagnosis of cognitive impairment was analyzed by receiver operating characteristic (ROC) curve with diagnostic sensitivity and specificity. Two-tailed $\mathrm{p}<0.05$ was considered statistically significant.

\section{Results}

\section{General characteristic of the two groups}

The general clinical characteristic and demographic features of the included PD-CI and PDN groups were shown in Table 1. There were significant statistical difference in the aspects of education level $(\mathrm{p}=0.02)$, MoCA score $(p<0.001)$, UPDRSIII score $(p<0.0001)$ and levodopa (LED) dosage $(p=0.004)$. 
Table 1: The clinical characteristics and demographic features of the included PD-Cl and PDN patients.

\begin{tabular}{llll}
\hline Characteristics & PD-Cl(n=41) & PDN(n=40) & p-value \\
\hline Gender[n,(\%)] & & & 0.56 \\
Male & $22(53.7)$ & $24(60.0)$ & \\
Female & $19(46.3)$ & $16(40.0)$ & \\
Diabetes[n,(\%)] & & & 0.49 \\
Positive & $5(12.2)$ & $3(7.5)$ & \\
Negative & $36(87.8)$ & $37(92.5)$ & \\
Age(year) & $63.3 \pm 12.3$ & $61.7 \pm 10.6$ & 0.53 \\
Education(year) & $6.2 \pm 4.1$ & $8.3 \pm 3.6$ & 0.02 \\
Course of disease (month) & $64.2 \pm 48.7$ & $57.4 \pm 41.6$ & 0.50 \\
UPDRS回 & $30.4 \pm 10.3$ & $20.6 \pm 8.9$ & $<0.0001^{*}$ \\
MoCA score & $18.2 \pm 4.3$ & $25.7 \pm 3.2$ & $<0.001$ \\
H\&Y stage & $2.5(2.0-3.0)$ & $2.0(1.5-2.5)$ & 0.06 \\
LED(mg/d) & $465.5 \pm 241.3$ & $312.4 \pm 214.6$ & $0.004^{*}$ \\
Ccr(ml/min) & $88.6 \pm 28.4$ & $92.1 \pm 25.3$ & 0.45 \\
Hypertension[n,(\%)] & $10(24.4)$ & $8(20.0)$ & 0.63 \\
Heart disease[n,(\%)] & $7(17.1)$ & $7(17.5)$ & 0.95 \\
Stroke[n,(\%)] & $3(7.3)$ & $4(10.0)$ & 0.67 \\
Hypercholesterinaemia[n,(\%)] & $12(29.3)$ & $10(25.0)$ & 0.67 \\
\hline & & & \\
& & & \\
\hline
\end{tabular}

LED: levodopa

Ccr: Creatinine clearance rate

${ }^{*} \mathrm{p}<0.05$ between PD-Cl and PDN groups

Table 2: The comparison of serum Hcy, folate, VitB12 between PD-Cl and PDN groups.

\begin{tabular}{llll}
\hline Characteristics & PD-Cl & PDN & p-value \\
\hline Hcy $(\mu \mathrm{mol} / \mathrm{L})$ & $21.7 \pm 6.2$ & $14.1 \pm 5.7$ & $<0.001$ \\
Folate $(\mathrm{ng} / \mathrm{mL})$ & $9.2 \pm 3.7$ & $12.4 \pm 4.5$ & 0.001 \\
VitB12 $(\mathrm{pg} / \mathrm{mL})$ & $354.1 \pm 123.5$ & $378.7 \pm 128.2$ & 0.38 \\
\hline
\end{tabular}

\section{Logistic regression analysis of independent factors for PD-CI}

The factors with statistical difference between PD-CI and PDN were further analyzed by logistic regression test to identify the independent factors for PD-CI. The results demonstrated that the MoCA and UPDRSIII score were independent factors for PD-CI $(\mathrm{p}<0.05)$.

\section{Serum Hcy, folate, VitB12}

The serum concentrations of Hcy, folate, VitB12 in both groups are given in Table 2. The serum level of Hcy in PD-CI group was significantly higher than that of PDN group $(p<0.05)$, but serum folate was significantly lower than PDN group $(\mathrm{p}<0.05)$, Figure 1.

\section{Diagnostic performance of serum}

The diagnostic sensitivity, specificity and AUC are shown in Table 3. 
A

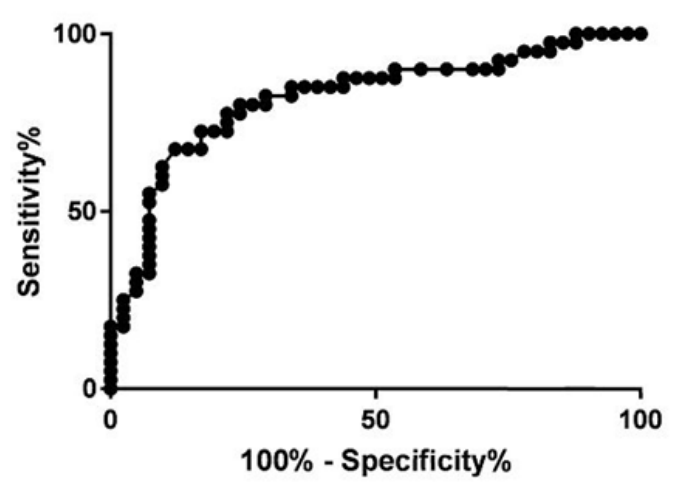

B

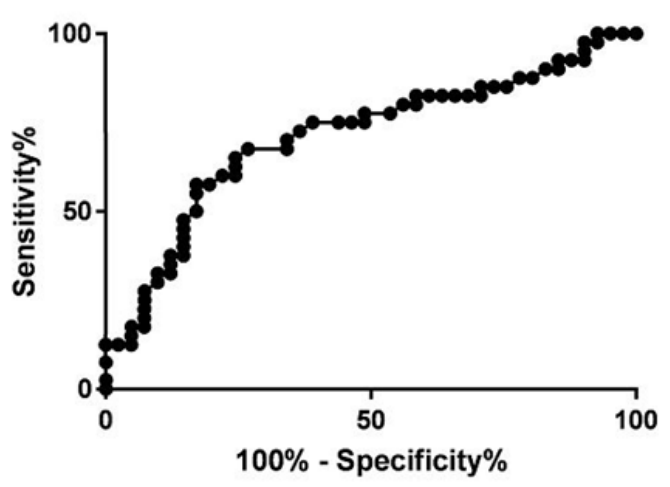

Figure 1: ROC curve of serum Hcy, folate in diagnosis of cognitive impairment in patients with Parkinson's disease(A: ROC curve for serum Hcy; B:ROC curve for serum folate).

Table 3: The diagnostic performance of serum Hcy and folate as serological marker for cognitive impairment in patients with Parkinson's disease.

\begin{tabular}{lll}
\hline Parameter & Hcy & Folate \\
\hline Sensitivity & $77.5 \%(95 \% \mathrm{Cl}: 61.6 \%-89.2 \%)$ & $72.5 \%(95 \% \mathrm{Cl}: 56.1 \%-85.4 \%)$ \\
Specificity & $78.1 \%(95 \% \mathrm{Cl}: 62.4 \%-89.4 \%)$ & $63.4 \%(95 \% \mathrm{Cl}: 46.9 \%-77.9 \%)$ \\
Cut off value & 17.2 & 9.5 \\
Likelihood ratio & 3.5 & 1.9 \\
AUC & $0.82(95 \% \mathrm{Cl}: 0.73-0.91)$ & $0.71(95 \% \mathrm{Cl}: 0060-0.83)$ \\
p-value & $<0.001$ & 0.001 \\
\hline
\end{tabular}

\section{Discussion}

In our present work, we included 81cases of PD and discussed the correlation of serum Hcy and folate concentration l with CI in PD patients. The results indicated that serum Hcy and folate were different in PD-CI and PDN patients which may play an essential role in cognitive impairment development in patients with PD and can be used as promising serological diagnostic marker. However, we also found that the LED dosage between PD-CI and PDN group were also statistical different, this may be a potential mixed risk factor for PD-CI. As known that cognitively impaired in patients of PD were significantly impaired in their motoric functioning (UPDRS III). In our work, the UPDRS III score of PD-CI group was significantly higher than of the PDN group.

Parkinson's disease (PD) is a common neurodegenerative disease, and its main pathological change is substantia nigra dopamine $[14,15]$. The degeneration and necrosis of dopamine (DA) neurons and the formation of misfolded alpha-synuclein protein are clinically manifested as typical motor symptoms such as retardation of movement, quiescent tremor, myotonia and postural gait disorder and non-motor symptoms (NMS) accompanied by cognitive impairment (CI), olfactory impairment, sensory impairment etc. [16-18]. Publications have shown that NMS throughout the whole process of disease progression in PD patients [19]. Independent clinical manifestations other than motor symptoms had their pathological basis [20, 21]. It is not a complication of motor symptoms itself, but usually affects the daily life of the patients. Especially in the early stage of disease, compared with motor symptoms, NMS has a greater and more serious impact on the quality of life of patients with PD, and cognitive impairment is a common NMS in patients with PD. However, the accurate mechanism of cognitive impairment in PD is not completely clear. Most of the studies believed that the main mechanism of cognitive impairment of PD patients is oxidative stress injury [22].

VitB12 is the only metal-containing vitamin, which is an important coenzyme for nucleotide synthesis in cells. VitB participates in deoxynucleic acid synthesis, fat, 
carbohydrate and protein metabolism. VitB participates in methyl transformation and folic acid metabolism in organisms, and improves folic acid utilization. Studies have shown that serum VitB12 level in patients with PD decreases and may be involved in the pathogenesis of PD. In this study, we found no significant difference in VitB12 between PD group and the control group. The reason may be related to the increase of oxidative stress in PD patients. However, oxidative stress could increase the demand of VitB. Immune system activation is a major cause of oxidative stress when a high amount of antimicrobial reactive oxygen species are released [23]. Increased formation of neopterin and enhanced degradation of tryptophan suggest activated cell-mediated immune response in patients with advanced Parkinson's disease [24].

Hcy is a derivative produced by demethylation of thiomethine which metabolized mainly through two pathways: methylation and transsulfuration. It has been reported that elevated plasma Hcy was associated with neurodegenerative diseases, such as cognitive impairment of PD patients, Alzheimer's disease and vascular dementia [25]. Zoccolella et al. [26] found that the level of Hcy in $\mathrm{CI}$ group was higher than that in normal group, and the decrease of cognitive function was closely related to the level of Hcy. Louis et al. [27] further confirmed that the development of MCI in PD patients was significantly correlated with the level of Hcy. In conclusion, significant differences of serum Hcy and folate were found between PD-CI and PDN patients. The serum level of Hcy and folate can be used as serological biomarker for predicting cognitively impairment in patients with PD.

Conflict of interest: Authors state no conflict of interest

\section{References}

1. Tysnes OB, Storstein A. Epidemiology of Parkinson's disease. J Neural Transm (Vienna) 2017;124:901-905.

2. Elbaz A, Carcaillon L, Kab S, Moisan F. Epidemiology of Parkinson's disease. Rev Neurol (Paris) 2016;172:14-26.

3. Mahajan A, Balakrishnan P, Patel A, Konstantinidis I, Nistal D, Annapureddy N, et al. Epidemiology of inpatient stay in Parkinson's disease in the United States: Insights from the Nationwide Inpatient Sample. J Clin Neurosci 2016;31:162-5.

4. Cali F, Cantone M, FII C, Lanza G, Ruggeri G, Chiavetta V, Salluzzo R, et al. Interpreting Genetic Variants: Hints from a Family Cluster of Parkinson's Disease. J Parkinsons Dis 2019;9:203-206.
5. Ezquerra M, Martí MJ, Fernández-Santiago R. Parkinson's disease as a systemic pathology. Aging (Albany NY) 2019;11:1081-1082.

6. Ikuno M, Yamakado H, Akiyama H, Parajuli LK, Taguchi K, Hara J, et al. GBA haploinsufficiency accelerates alpha synuclein pathology with altered lipid metabolism in a prodromal model of Parkinson's disease $=$. Hum Mol Genet 2019.

7. Guo Y, Xu W, Liu FT, Li JQ, Cao XP, Tan L, et al. Modifiable risk factors for cognitive impairment in Parkinson's disease: A systematic review and meta-analysis of prospective cohort studies. Mov Disord 2019.

8. Nicoletti A, Luca A, Baschi R, Cicero CE, Mostile G, Davì M, et al. Incidence of Mild Cognitive Impairment and Dementia in Parkinson's Disease: The Parkinson's Disease Cognitive Impairment Study. Front Aging Neurosci 2019;11:21.

9. Vasconcellos LFR, Pereira JS, Charchat-Fichman H, Greca D, Cruz M, Blum AL, et al. Mild cognitive impairment in Parkinson's disease: Characterization and impact on quality of life according to subtype. Geriatr Gerontol Int 2019.

10. Martinez-Horta S, Kulisevsky J. Mild cognitive impairment in Parkinson's disease. J Neural Transm (Vienna) 2019.

11. Ebrahimpour A, Vaghari-Tabari M, Qujeq D, Moein S, Moazezi Z. Direct correlation between serum homocysteine level and insulin resistance index in patients with subclinical hypothyroidism: Does subclinical hypothyroidism increase the risk of diabetes and cardio vascular disease together. Diabetes Metab Syndr 2018;12:863-867.

12. Postuma RB, Berg D, Stern M, Poewe W, Olanow CW, Oertel W, et al. MDS clinical diagnostic criteria for Parkinson's disease. Mov Disord 2015;30:1591-601.

13. Li J, Jin M, Wang L, Qin B, Wang K. MDS clinical diagnostic criteria for Parkinson's disease in China. J Neurol 2017;264:476-481.

14. Haddad F, Sawalha M, Khawaja Y, Najjar A, Karaman R. Dopamine and Levodopa Prodrugs for the Treatment of Parkinson's Disease. Molecules 2017;23.

15. Warren N, O'Gorman C, Lehn A, Siskind D. Dopamine dysregulation syndrome in Parkinson's disease: a systematic review of published cases. J Neurol Neurosurg Psychiatry 2017;88:1060-1064.

16. Dulski J, Schinwelski M, Konkel A, Grabowski K, Libionka W, Wąż P, et al. The impact of subthalamic deep brain stimulation on sleep and other non-motor symptoms in Parkinson's disease. Parkinsonism Relat Disord 2019.

17. Aldaz T, Nigro P, Sánchez-Gómez A, Painous C, Planellas L, Santacruz P, et al. Non-motor symptoms in Huntington's disease: a comparative study with Parkinson's disease. J Neurol 2019.

18. Suzuki K, Fujita H, Matsubara T, Hirata K. Non-motor symptoms in postural instability/gait difficulty subtype in the early stage of Parkinson's disease. Eur J Neurol 2019;26:e37.

19. Durcan R, Wiblin L, Lawson RA, Khoo TK, Yarnall AJ, Duncan GW, et al. Prevalence and duration of non-motor symptoms in prodromal Parkinson's disease. Eur J Neurol 2019.

20. Rosqvist K, Odin P, Hagell P, Iwarsson S, Nilsson MH, Storch A. Dopaminergic Effect on Non-Motor Symptoms in Late Stage Parkinson's Disease. J Parkinsons Dis 2018.

21. Matsubara T, Suzuki K, Fujita H, Watanabe Y, Sakuramoto H, Matsubara M, et al. Autonomic Symptoms Correlate with Non-Autonomic Non-Motor Symptoms and Sleep Problems in 
Patients with Parkinson's Disease. Eur Neurol 2018;80:193-

199.

22. Cheung C, Bhimani R, Wyman JF, Konczak J, Zhang L, Mishra $\mathrm{U}$, et al. Effects of yoga on oxidative stress, motor function, and non-motor symptoms in Parkinson's disease: a pilot randomized controlled trial. Pilot Feasibility Stud 2018;4:162.

23 Widner B, Leblhuber F, Frick B, Laich A, Artner-Dworzak E, Fuchs D. Moderate hyperhomocysteinaemia and immune activation in Parkinson's disease. J Neural Transm (Vienna) 2002;109:1445-

52.

24 Widner B, Leblhuber F, Fuchs D. Increased neopterin production and tryptophan degradation in advanced Parkinson's disease. J Neural Transm (Vienna) 2002;109:181-9.

25. Huiyun Li LL, Fangying Zhou TZ. Elevated ESRS, serum FIB, Hcy and stroke history were independent risk factors to PAIS. Pteridines 2018;29:165-171.

26. Zoccolella S, Lamberti SV, lliceto G, Santamato A, Lamberti $P$, Logroscino G. Hyperhomocysteinemia in L-dopa treated patients with Parkinson's disease: potential implications in cognitive dysfunction and dementia. Curr Med Chem 2010;17:3253-61.

27. Louis ED, Schupf N, Tang MX, Marder K, Luchsinger JA. Mild parkinsonian signs and plasma homocysteine concentration in community-dwelling elderly individuals. Arch Neurol 2007;64:1646-51. 